\title{
Microwave-assisted hydrothermal synthesis of highly luminescent ZnSe-based quantum dots with a quantum yield higher than $90 \%$
}

Yong-Shin Lee, Kaoru Nakano, Hang-Beom Bu, Dae Gwi Kim

\begin{tabular}{|c|l|}
\hline Citation & Applied Physics Express, 10(6);065001 \\
\hline Issue Date & 2017-05-11 \\
\hline Type & Journal Article \\
\hline Textversion & Author \\
\hline Rights & $\begin{array}{l}\text { C 2017 The Japan Society of Applied Physics. This is an author-created, } \\
\text { un-copyedited version of an article accepted for publication in Applied Physics } \\
\text { Express. The publisher is not responsible for any errors or omissions in this version } \\
\text { of the manuscript or any version derived from it. The Version of Record is available } \\
\text { online at https://doi.org/10.7567/APEX.10.065001. }\end{array}$ \\
\hline DOI & 10.7567/APEX.10.065001 \\
\hline
\end{tabular}

Self-Archiving by Author(s)

Placed on: Osaka City University

LEE, Y.-S., NAKANO, K., BU, H.-B., \& KIM, D. G. (2017). Microwave-assisted hydrothermal synthesis of highly luminescent ZnSe-based quantum dots with a quantum yield higher than $90 \%$. Applied Physics Express. 10, 065001. 
Microwave-assisted hydrothermal synthesis of highly luminescent ZnSe-based quantum dots with a quantum yield higher than $90 \%$

Yong-Shin Lee, Kaoru Nakano, Hang-Beom Bu, and DaeGwi Kim*

Department of Applied Physics, Graduate School of Engineering, Osaka City University

3-3-138, Sugimoto, Sumiyoshi-ku, Osaka 558-8585, Japan

E-mail: tegi@a-phys.eng.osaka-cu.ac.jp

Highly luminescent ZnSe-based quantum dots (QDs) were synthesized by a microwave-assisted hydrothermal method. The characteristics of the $\mathrm{ZnSe}$ precursor solution strongly influenced the photoluminescence (PL) quantum yields (QYs) of the QDs. The PL QY of ZnSe-core QDs synthesized under the optimum conditions reached $60 \%$. Furthermore, the PL QY further increased to higher than $90 \%$ when a $\mathrm{ZnS}$ shell was applied to prepare ZnSe/ZnS-core/shell QDs. 
In semiconductor quantum dots (QDs), the quantum nature of electrons and/or excitons that cannot be observed in bulk crystals becomes apparent because of QD's finite size. ${ }^{1-3)}$ A well-known example is quantum size effects. ${ }^{1-3)}$ When an electron is confined to small spaces with dimensions of the order of several nanometers, the eigenstate of excitons is discretized and the exciton energy changes in accordance with a change in the QD size. ${ }^{1,4)}$ Thus, absorption and photoluminescence (PL) energies can be controlled by manipulating QD's size. Because the optical properties of semiconductor QDs strongly depend on their size, they have been intensively investigated from the viewpoint of basic science to elucidate the size dependence of their physical and/or chemical properties. ${ }^{5-8)}$

In addition, numerous efforts have been made to apply these characteristics of QDs to devices such as light-emitting diodes (LEDs) ${ }^{9,10)}$ and QD lasers. ${ }^{11)}$ Furthermore, because semiconductor QDs exhibit less photodegradation than organic molecules, QDs have potential applications as fluorescent markers. ${ }^{7,12)}$ Therefore, semiconductor QDs with a high PL quantum yield (QY) are essential with respect to the application of semiconductor QDs in optoelectronic devices.

Colloidal semiconductor QDs have been synthesized using various methods. ${ }^{13-19)}$ The representative method is hot-injection method of promoting crystal growth via high-temperature heating in an organic solvent. ${ }^{13-15)}$ Using this method, researchers have prepared numerous II-VI semiconductor QDs, e. g., CdSe with a high PL QY (higher than 50\%). ${ }^{14,15)}$ However, from an environment and application viewpoint, the hot-injection method, which uses organic solvents, and the presence of $\mathrm{Cd}$, which is toxic, have raised concerns.

ZnSe QDs have attracted significant attention in recent years. ${ }^{16-20)}$ However, 
compared with the number of reports related to the synthesis and characterization of $\mathrm{CdSe}$, the number of reports related to $\mathrm{ZnSe} \mathrm{QDs}$ is small. ZnSe is a wide-gap semiconductor with a 2.7-eV band-gap in bulk crystals. Thus far, ZnSe QDs have been synthesized in both organic solvents ${ }^{16,17)}$ and aqueous solutions. ${ }^{18-20)}$ Qian et al. proposed a simple and easy method of preparing ZnSe QDs through microwave irradiation of aqueous solutions ${ }^{18)}$; they prepared alloyed ZnSe-ZnS QDs with a QY of $17 \%$ using mercaptopropionic acid (MPA) as a ligand. This method has the advantage of enabling the rapid synthesis of QDs; however, the reported QY of the obtained QDs is low. Fang et al. reported the preparation of $\mathrm{ZnSe} / \mathrm{ZnS}$-core/shell QDs with a QY of $64 \%$ using a two-step process in which the researchers prepared the core and subsequently added a shell precursor in which glutathione ( $\gamma$-glutamyl-cysteinyl-glycine; GSH) was used as a ligand. $\left.{ }^{19}\right)$ Zhao et al. prepared ZnSe/ZnS-core/shell QDs with a QY of $39 \%$ in a single step through thermal decomposition of the ligand N-acetyl-L-cysteine (NAC). ${ }^{20)}$ However, the PLQY was lower than that of CdSe and CdTe QDs, thus, scope for further improvements in the preparation condition exists.

In ref. 20, a study of $\mathrm{pH}$ conditions of precursor solutions was conducted for $\mathrm{pH}$ levels between 9.0 and 10.5. Recently, we reported the hydrothermal synthesis of NAC-capped ZnSe QDs without shell structure. ${ }^{21)}$ We found that the PL intensity of the ZnSe QDs was strongly dependent on the $\mathrm{pH}$ of the precursor solutions and that the optimum $\mathrm{pH}$ value was $\sim 6 .^{21)}$ According to the aforementioned reports, high-quality ZnSe QDs can be prepared through a systematic investigation of the preparation conditions of the precursor solutions, such as their $\mathrm{pH}$ value and the molar ratio among the $\mathrm{Zn}$-ion source, Se-ion source, and the ligand. 
In the present work, we report the synthesis of highly luminescent ZnSe-core and ZnSe/ZnS-core/shell QDs. By optimizing the preparation conditions, we synthesized ZnSe-core QDs with a high QY of 60\%. Moreover, after the ZnS-shell coating was applied, the QY was further increased to higher than $90 \%$, which, to our knowledge, is the highest QY reported thus far for ZnSe-based QDs.

The ZnSe QDs were synthesized using a microwave-assisted hydrothermal method. A precursor solution was prepared according to the procedure reported by ref. 21 . Briefly, $\mathrm{ZnCl}_{2}(2.0 \mathrm{mmol})$ and $\mathrm{NAC}(4.8 \mathrm{mmol})$ were dissolved in $100 \mathrm{~mL}$ of deionized (DI) water followed by adjustment to $\mathrm{pH}$ 8.5. A freshly prepared NaHSe solution was then added. Finally, the precursor solution was adjusted to $\mathrm{pH}$ 6.0. To deduce the optimum conditions, we prepared various precursor solutions with different molar ratios of $\mathrm{Zn}^{2+}, \mathrm{Se}^{2-}$, and NAC. The influence of the $\mathrm{pH}$ shortly before the addition of the NaHSe solution on the PL intensity was also investigated. ZnSe QDs were synthesized under microwave irradiation for $4 \mathrm{~min}$ at $180^{\circ} \mathrm{C}$ using a single mode CEM Discover system.

Absorption and PL spectra were recorded using a JASCO V-650 UV/Vis spectrophotometer and a JASCO FP-8300 spectrofluorometer, respectively. PL QYs were measured with an absolute PL measurement system (Hamamatsu, CQQ20). X-ray diffraction (XRD) measurements were performed on a Rigaku SmartLab equipped with a $\mathrm{Cu} \mathrm{K} \alpha$ radiation source $(\lambda=0.154 \mathrm{~nm})$.

Figure 1 shows the PL spectra of $\mathrm{ZnSe}$ QDs synthesized with $\mathrm{Zn}^{2+}: \mathrm{Se}^{2-}: \mathrm{NAC}=1.0$ : 0.3: 2.4. The $\mathrm{pH}$ value shortly before the addition of the NaHSe solution was 8.5 . The band-edge PL is clearly observed in the spectra of all of the samples. With an increase in the heating time, the intensity ratio of the defect-related PL to the band-edge PL 
decreased; the ZnSe QDs synthesized with a reaction time of 3 min exhibited the highest PL intensity.

Wei et al. reported that in the synthesis of CdSe QDs through a hydrothermal method, the $\mathrm{Cd}^{2+}: \mathrm{Se}^{2-}:$ NAC molar ratio strongly affected the PL intensity of the CdSe QDs. ${ }^{22)}$ Thus, we synthesized ZnSe QDs under various conditions by changing the molar ratio of $\mathrm{Zn}^{2+}$ to $\mathrm{Se}^{2-}\left(\mathrm{Zn}^{2+}: \mathrm{Se}^{2-}=1.0: X\right.$, where $\left.X=0.1,0.2,0.3,0.4\right)$ and that of $\mathrm{Zn}^{2+}$ to $\mathrm{NAC}\left(\mathrm{Zn}^{2+}: \mathrm{NAC}=1.0: Y\right.$, where $\left.1.2 \leq Y \leq 6.0\right)$. Figure 2 shows the experimental results for the dependence of the PL QY on the $\mathrm{Zn}^{2+}: \mathrm{Se}^{2-}: \mathrm{NAC}$ molar ratio. The results reveal that the $\mathrm{ZnSe}$ QDs synthesized with $\mathrm{Zn}^{2+}: \mathrm{Se}^{2-}: \mathrm{NAC}=1.0: 0.2: 2.4$ exhibited the highest PL intensity.

Next, we focused on the dependence of the PL intensity of ZnSe QDs on the pH shortly before the addition of the NaHSe solutions. The $\mathrm{pH}$ before the addition of the NaHSe solutions was changed from 6.0 to 12.0 . The $\mathrm{Zn}^{2+}: \mathrm{Se}^{2-}$ : NAC molar ratio and the final $\mathrm{pH}$ of the $\mathrm{ZnSe}$ precursor solution were fixed at 1.0: $0.2: 2.4$ and 6.0, respectively. According to the theory for the quantum confinement effect in QDs, ${ }^{23)}$ the lowest exciton energy in a spherical QD with a radius $R$ is given by

$$
E(R)=E_{g}+\frac{\eta^{2}}{2 \mu}\left(\frac{\pi}{R}\right)^{2}-1.8 \frac{e^{2}}{\varepsilon R}
$$

where $E_{g}, \mu$, and $\varepsilon$ are the band-gap energy, reduced mass, and dielectric constant, respectively. By substituting the values in a ZnSe bulk crystal for each parameter, eq. (1) can be transformed as follows, which corresponds to eq. (4) in ref. 24,

$$
E(d)=2.67+\frac{11.9}{d^{2}}-\frac{0.596}{d}(\mathrm{eV})
$$

where diameter $(d)$ is in nanometers. The average diameter of ZnSe QDs was calculated from the absorption peak energy based on eq. (2). As shown in Fig. 3, the PL QYs of 
ZnSe QDs were strongly dependent on the $\mathrm{pH}$ before the addition of the NaHSe solution. The experimental results clearly indicate that the $\mathrm{pH}$ value before the addition of the NaHSe solutions is an important factor and that its optimum value is 8.5 . Thus, we concluded that the optimum conditions for the synthesis of $\mathrm{ZnSe}$ QDs are a $\mathrm{Zn}^{2+}$ : $\mathrm{Se}^{2-}$ : NAC molar ratio of 1.0: $0.2: 2.4$, a $\mathrm{pH}$ value of 8.5 shortly before the addition of the NaHSe solution, and a final $\mathrm{pH}$ of the precursor solution is 6.0. The highest obtained QY was $60 \%$, as far as we know, this QY is the highest value reported thus far for water-soluble ZnSe QDs.

As pointed out in ref. 20, microwave-assisted hydrothermal synthesis requires professional and expensive microwave equipment. To verify whether the optimum conditions deduced in the present work are also applicable in a conventional hydrothermal synthesis, we attempted to synthesize ZnSe QDs using a hydrothermal method without microwave irradiation. Notably, ZnSe QDs obtained by heating the precursor solution at $200^{\circ} \mathrm{C}$ for 60 min using an autoclave also exhibited a high PL QY of $60 \%$, which matches that of the QDs synthesized using microwave irradiation. Thus, the preparation conditions of the ZnSe precursor solution strongly influence the quality of the obtained ZnSe QDs, whereas the heating method (i.e., with/without microwave irradiation) does not.

In the next step, to further improve the QY, we synthesized $\mathrm{ZnSe} / \mathrm{ZnS}$-core/shell QDs. When $\mathrm{ZnS}$ is used as a shell, ZnSe/ZnS forms a type-I potential structure in which electrons and holes are confined in the $\mathrm{ZnSe}$ core. ${ }^{25)}$ Furthermore, because the non-radiative recombination process caused by the surface state of $\mathrm{ZnSe}$ QDs is suppressed, an increase in the PL intensity is expected. ZnSe/ZnS-core/shell QDs were prepared as follows. First, $490 \mathrm{mg}$ of $\mathrm{Na}_{2} \mathrm{~S} \cdot 9 \mathrm{H}_{2} \mathrm{O}$ was added to $20 \mathrm{~mL}$ of DI water to 
prepare the $\mathrm{S}^{2-}$-ion source. NAC (1.96 g) was then dissolved in $100 \mathrm{~mL}$ of DI water, and $745 \mathrm{mg}$ of $\mathrm{Zn}\left(\mathrm{ClO}_{4}\right)_{2} \cdot 6 \mathrm{H}_{2} \mathrm{O}$ was added as a $\mathrm{Zn}^{2+}$-ion source. $\mathrm{NaOH}$ was added to this solution to adjust the $\mathrm{pH}$ to 5.0 , and then $6 \mathrm{~mL}$ of the $\mathrm{S}^{2-}$-ion source was added. Finally, the $\mathrm{ZnS}$ precursor solution was prepared by adding diluted $\mathrm{HCl}$ to adjust the $\mathrm{pH}$ to 6.0 . The $\mathrm{Zn}^{2+}: \mathrm{S}^{2-}$ : NAC molar ratio was $1.0: 0.3: 4.8$. The $\mathrm{ZnS}$ precursor solution was added to the already prepared $\mathrm{ZnSe}$ QD solution, and ZnSe/ZnS-core/shell QDs were synthesized by microwave irradiation.

Figure 4 shows the absorption spectra of ZnSe-core and ZnSe/ZnS-core/shell QDs. A new absorption feature was confirmed at $4.4 \mathrm{eV}$, which is a higher-energy region than the band-gap energy of $\sim 3.7 \mathrm{eV}$ in a $\mathrm{ZnS}$ bulk crystal; this feature was not observed in the spectra of the ZnSe-core QDs. It is well known that an additional absorption structure is observed on the high energy side than the band-gap energy of shell material when core/shell-QDs are formed. ${ }^{26)}$ This transition originates from the higher excited states of electrons and holes spreading over entire core/shell-QDs. Furthermore, the absorption peak the ZnSe/ZnS-core/shell QDs shifted toward lower energies compared to the absorption peak of the ZnSe-core QDs. Conceivably, the effective confinement energy of the wave function may have been decreased when the ZnSe QDs were covered with $\mathrm{ZnS}{ }^{25)}$ Thus, these observations suggest that the ZnSe/ZnS-core/shell QDs were successfully synthesized.

To confirm the formation of the core/shell structure, X-ray structural analyses of both the $\mathrm{ZnSe}$-core and the $\mathrm{ZnSe} / \mathrm{ZnS}$-core/shell QDs were carried out; the corresponding XRD patterns are shown in Fig. 5. The three broad diffraction peaks in the pattern of the ZnSe-core QDs correspond to the (111), (220), and (311) planes of the cubic zinc-blende ZnSe lattice structure (JCPDS Card No. 80-0021). Compared with the 
peaks in the XRD pattern of the ZnSe-core QDs, those of the ZnSe/ZnS-core/shell QDs were shifted to higher $2 \theta$ angles. Usually, when core/shell-QDs are formed, the diffraction peak is shifted to that of the shell material. ${ }^{19,20)}$ Since the lattice constant of $\mathrm{ZnS}$ is $5.40 \AA$, which is smaller than $5.67 \AA$ in $\mathrm{ZnSe}$, the diffraction peak of $\mathrm{ZnS}$ appears higher $2 \theta$ angles than those of $\mathrm{ZnSe}$. On the basis of the experimental results in Figs. 4 and 5, we concluded that $\mathrm{ZnSe} / \mathrm{ZnS}$-core/shell QDs were synthesized successfully.

Finally, we investigated the PL QY of the ZnSe/ZnS-core/shell QDs. Figure 6 shows the PL spectra of the ZnSe-core and the ZnSe/ZnS-core/shell QDs. Compared with the PL intensity of the ZnSe-core QDs, that of ZnSe/ZnS-core/shell QDs is higher. Notably, ZnSe/ZnS-core/shell QDs exhibit a PL QY higher than 90\%.To the best of our knowledge, this PL QY is the highest reported thus far for ZnSe-based QDs.

In summary, we have investigated the optimum conditions for the synthesis of highly luminescent ZnSe QDs. The characteristics of the ZnSe precursor solution strongly influenced PL QYs of the ZnSe QDs. The optimum conditions for the synthesis of $\mathrm{ZnSe}$ QDs were found to be (a) a $\mathrm{Zn}^{2+}: \mathrm{Se}^{2-}$ : NAC molar ratio of 1.0: 0.2: 2.4, (b) a $\mathrm{pH}$ shortly before the addition of the NaHSe solution of 8.5, and (c) a final $\mathrm{pH}$ of the precursor solution of 6.0. The PL QY of the ZnSe-core QDs synthesized under the optimum conditions reached $60 \%$. By contrast, with the synthesis of ZnSe/ZnS-core/shell QDs, the PL QY was increased to higher than 90\%. The present results open the possibility of using ZnSe-based QDs as blue luminescence materials. 
1) L. Brus, Appl. Phys. A 53, 465 (1991).

2) Y. Wang and N. Herron, J. Phys. Chem. 95, 525 (1991).

3) Y. Kayanuma, Phys. Rev. B 38, 9797 (1988).

4) L. E. Brus, J. Chem. Phys. 80, 4403 (1984).

5) D. J. Norris and M. G. Bawendi, Phys. Rev. B 53, 16338 (1996).

6) D. J. Norris, A. L. Efros, M. Rosen, and M. G. Bawendi, Phys. Rev. B 53, 16347 (1996).

7) X. michalet, F. F. Pinaud, L. A. Bentolila, J. M. Tsay, S. Doose, J. J. Li, G. Sundaresan, A. M. Wu, S. S. Gambhir, and S. Weiss, Science 307, 538 (2005).

8) A. P. Alivisatos, Science 271, 933 (1996).

9) D. V. Talapin and J. Steckel, MRS Bull. 38, 685 (2013).

10) Y.Shirasaki, G. J. Supran, M. G. Bawendi, and V. Bulović, Nat. Photonics 7, 13 (2013).

11) V. I. Klimov, A. A. Mikhailovsky, S. Xu, A. Malko, J. A. Hollingsworth, C. A. Leatherdale, H.-J. Eisler, and M. G. Bawendi, Science 290, 314 (2000).

12) Q. Wang, Y. Xu, X. Zhao, Y. Chang, Y. Liu, L. Jiang, J. Sharma, D.-K. Seo, and H. Yan, J. Am. Chem. Soc. 129, 6380 (2007).

13) C. B. Murray, D. J. Norris, and M. G. Bawendi, J. Am. Chem. Soc. 115, 8706 (1993).

14) D. V. Talapin, A. L. Rogach, A. Kornowski, M. Haase, and H. Weller, Nano Lett. 1, 207 (2001).

15) L. Qu and X. Peng, J. Am. Chem. Soc. 124, 2049 (2002).

16) M. A. Hines and P. G.-Sionnest, J. Phys. Chem. B 102, 3655 (1998).

17) P. D. Cozzoli, L. Manna, M. L. Curri, S. Kudera, C. Giannini, M. Striccoli, and A. 
Agostiano, Chem. Mater. 17, 1296 (2005).

18) H. Qian, X. Qiu, L. Li, and J. Ren, J. Phys. Chem. B 110, 9034 (2006).

19) Z. Fang, Y. Li, H. Zhang, X. Zhong, and L. Zhu, J. Phys. Chem. C 113, 14145 (2009).

20) D. Zhao, J.-T. Li, F. Gao, C. Zhang, and Z. He, RSC Adv. 4, 47005 (2013).

21) Y.-S. Lee, H.-B. Bu, T. Taniguchi, T. Takagi, S. Sobue, H. Yamada, T. Iwaki, and D. Kim, Chem. Lett. 45, 878 (2016).

22) C. Wei, J. Li, F. Gao, S. Guo, Y. Zhou, and D. Zhao, J. Spectroscopy 2015, 369145 (2015).

23) L. Brus, J. Phys. Chem. 90, 2555 (1986).

24) Z. Deng, F. L. Lie, S. Shen, I. Ghosh, M. Mansuripur, and A. J. Muscat, Langmuir 25, 434 (2009).

25) M. Lomascolo, A. Cretì, G. Leo, and L. Vasanelli, Appl. Phys. Lett. 82, 418 (2003).

26) X. Peng, M. C. Schlamp, A. V. Kadavanich, and A. P. Alivisatos, J. Am. Chem. Soc. 119, 7019 (1997). 


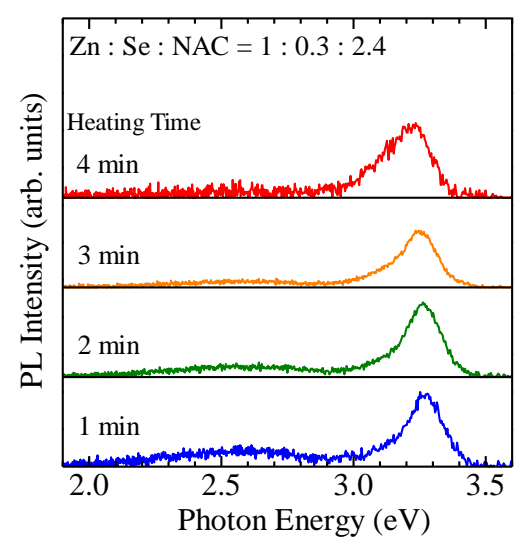

Fig. 1. PL spectra of ZnSe QDs synthesized with $\mathrm{Zn}^{2}: \mathrm{Se}^{2-}: \mathrm{NAC}=1.0: 0.3: 2.4$. The $\mathrm{pH}$ shortly before the addition of the NaHSe solution was 8.5. 


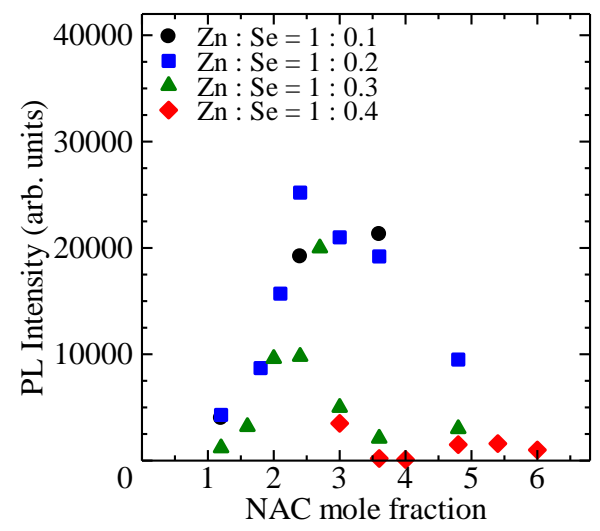

Fig. 2. The dependence of PL intensity on the $\mathrm{Zn}^{2+}: \mathrm{Se}^{2-}:$ NAC molar ratio. 


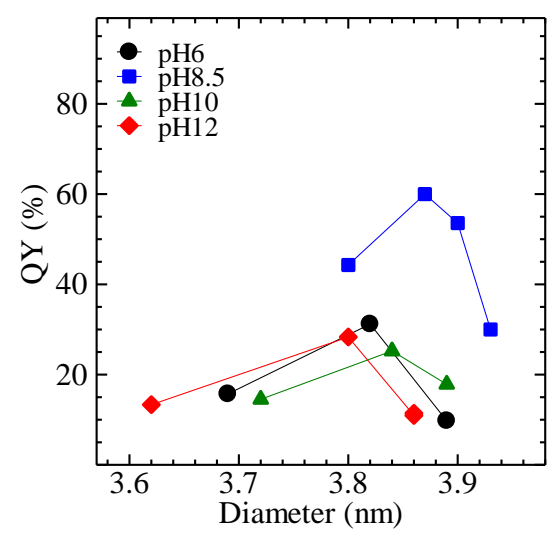

Fig. 3. The dependence of PL QY on the $\mathrm{pH}$ shortly before the addition of the NaHSe solutions. The $\mathrm{pH}$ before the addition of the NaHSe solutions was changed from 6.0 to 12.0. The $\mathrm{Zn}^{2+}: \mathrm{Se}^{2-}: \mathrm{NAC}$ molar ratio and the final $\mathrm{pH}$ of the $\mathrm{ZnSe}$ precursor solution were fixed at 1.0: 0.2: 2.4 and 6.0, respectively. 


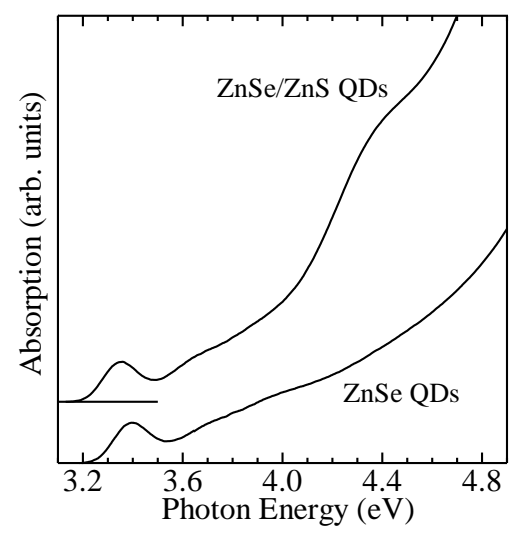

Fig. 4. Absorption spectra of ZnSe-core and ZnSe/ZnS-core/shell QDs. 


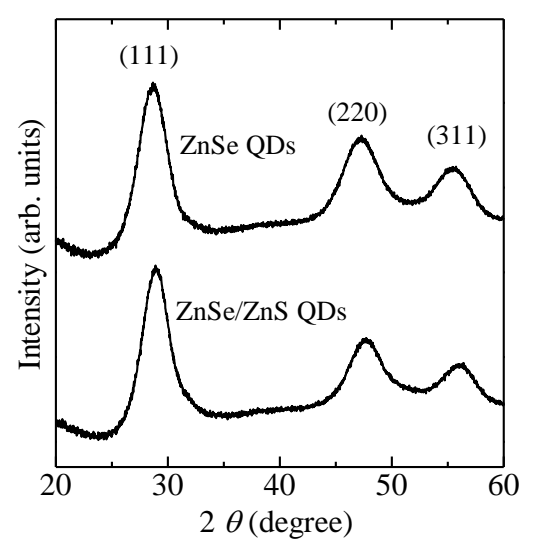

Fig. 5. X-ray diffraction patterns of ZnSe-core and ZnSe/ZnS-core/shell QDs. 


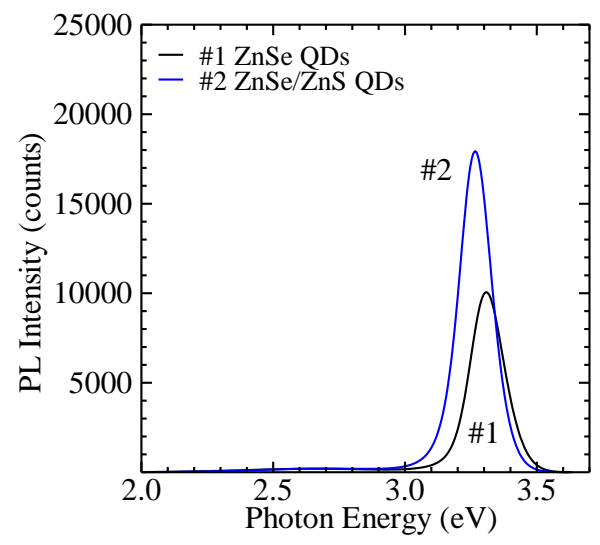

Fig. 6. PL spectra of ZnSe-core and ZnSe/ZnS-core/shell QDs. 